\title{
Investigating the bacterial microbiota of traditional fermented dairy products using propidium monoazide with single-molecule real-time sequencing
}

\author{
Lanxin Mo, ${ }^{*}$ Jie Yu, ${ }^{*}$ Hao Jin, Qiangchuan Hou, Caiqing Yao, Dongyan Ren, Xiaona An, \\ Tsedensodnom Tsogtgerel, and Heping Zhang† \\ Key Laboratory of Dairy Biotechnology and Engineering, Ministry of Education, Key Laboratory of Dairy Products Processing, \\ Ministry of Agriculture, Inner Mongolia Agricultural University, Huhhot 010018, P.R. China
}

\begin{abstract}
Traditional fermented dairy foods have been the major components of the Mongolian diet for millennia. In this study, we used propidium monoazide (PMA; binds to DNA of nonviable cells so that only viable cells are enumerated) and single-molecule real-time sequencing (SMRT) technology to investigate the total and viable bacterial compositions of 19 traditional fermented dairy foods, including koumiss from Inner Mongolia (KIM), koumiss from Mongolia (KM), and fermented cow milk from Mongolia (CM); sample groups treated with PMA were designated PKIM, PKM, and PCM. Full-length 16S rRNA sequencing identified 195 bacterial species in 121 genera and 13 phyla in PMA-treated and untreated samples. The PMA-treated and untreated samples differed significantly in their bacterial community composition and $\alpha$-diversity values. The predominant species in KM, KIM, and CM were Lactobacillus helveticus, Streptococcus parauberis, and Lactobacillus delbrueckii, whereas the predominant species in PKM, PKIM, and PCM were Enterobacter xiangfangensis, Lactobacillus helveticus, and E. xiangfangensis, respectively. Weighted and unweighted principal coordinate analyses showed a clear clustering pattern with good separation and only minor overlapping. In addition, a pure culture method was performed to obtain lactic acid bacteria resources in dairy samples according to the results of SMRT sequencing. A total of $102 \mathrm{LAB}$ strains were identified and $L b$. helveticus $(68.63 \%)$ was the most abundant, in agreement with SMRT sequencing results. Our results revealed that the bacterial communities of traditional dairy foods are complex and vary by type of fermented dairy product. The PMA treatment induced significant changes in bacterial community structure.
\end{abstract}

Received September 26, 2018.

Accepted January 14, 2019.

*These authors contributed equally to this work.

$\dagger$ Corresponding author: hepingdd@vip.sina.com
Key words: bacterial diversity, single molecule realtime (SMRT) sequencing, propidium monoazide, traditional fermented dairy product

\section{INTRODUCTION}

Homemade dairy foods are popular with the nomadic people of Inner Mongolia, China, and Mongolia, due to the abundance of raw milk. Traditional fermented dairy foods have been produced for millennia and consumed across the generations (Gesudu et al., 2016; Wang et al., 2016). Traditional fermented mare milk (koumiss, also known as airag and chige) and traditional fermented cow milk constitute a large part of the daily diet of Mongolian herdsmen. Koumiss is made from fresh mare milk using natural starters from the previous batch of koumiss and fermented in wooden casks or porcelain urns (Gesudu et al., 2016). The raw milk is anaerobically fermented at ambient temperature for 1 to $3 \mathrm{~d}$ and stirred using a wooden stick. Koumiss and traditional fermented cow milk are rich in natural microbial communities, which are determinants of their texture, fragrance, and health benefits (Li et al., 2017). The microbial communities, particularly lactic acid bacteria (LAB), present in koumiss and traditional fermented cow milk contribute to their quality and nutritional properties (e.g., appearance, taste, and flavor), but not to their shelf life (Sun et al., 2014). Thus, information on the bacterial community composition of fermented dairy foods would enable improvement of their flavor and texture.

Culture-based methods are the gold standard for detection and isolation of microorganisms. However, the pure culture method is laborious, and some fastidious microbes are not culturable because their natural growth conditions cannot be simulated (Oliver, 2005). A recently developed chromogenic culture medium enables the enumeration of some LAB species but cannot provide information on the composition of the bacterial communities in dairy foods (Galat et al., 2016). Therefore, novel methods for the detection and characterization of microbes in dairy products are required. 
The third-generation single-molecule real-time (SMRT) sequencing technology (Pacific Biosciences, Menlo Park, CA) enables comprehensive analysis of the microbial profiles of environmental samples based on the full-length bacterial 16S rRNA gene (Amir et al., 2013; Hou et al., 2015). To date, next-generation DNA sequencing techniques, such as 454 pyrosequencing and the Illumina platform (Illumina Inc., San Diego, CA), have been used to assess the microbial composition, including nonculturable taxa, of environmental samples. However, these technologies introduce bias because they partially sequence the $16 \mathrm{~S}$ rRNA gene (Cai et al., 2013; Klindworth et al., 2013). The Pacific Biosciences (PacBio) sequencing based on the full-length bacterial 16S rRNA gene has a higher taxonomic resolution than the in silico-generated PacBio bacterial 16S rRNA V4 gene, and the Illumina bacterial $16 \mathrm{~S}$ rRNA V4 gene is unable to identify closely related species compared with the PacBio full-length bacterial 16S rRNA gene (Singer et al., 2016). PacBio SMRT sequencing has been used to evaluate the safety of UHT milk and infant formula and to assess the bacterial communities of traditional artisanal cheeses and koumiss (Hou et al., 2015; Li et al., 2017; Jin et al., 2018). PacBio SMRT sequencing enables comprehensive and high-throughput analysis of microbial ecology at the species level based on fulllength 16S rRNA gene sequences.

Despite these advantages, PacBio SMRT technology is unable to reflect the presence of bacterial subpopulations in different viability states. This limitation can be overcome by using propidium monoazide (PMA), a DNA-binding fluorescent dye that penetrates only the compromised membranes of nonviable cells. The binding of PMA to DNA is made permanent by photolysis and the DNA cannot be amplified by PCR (Nocker, et al., 2006, 2010). Erkus et al. (2016) selectively profiled the viable microbial community during cheese ripening using PMA with metagenome sequencing. The PMA treatment did not limit the sequencing of total DNA, illustrating the usefulness of the 2-step protocol in complex ecosystems. To our knowledge, few studies have used PMA treatment in conjunction with PacBio SMRT sequencing. Thus, we applied these technologies to investigate the viable and nonviable bacterial communities of traditional dairy products to gain a deeper understanding of the bacterial population.

\section{MATERIALS AND METHODS}

\section{Sampling}

We investigated 19 samples of traditional fermented dairy products, comprising 12 koumiss samples from Inner Mongolia, China (KIM; KIM1 to KIM12), and
4 koumiss (KM; KM1 to KM4) and 3 fermented cow milk (CM; CM1 to CM3) samples from Selenge and Arkhangai, Bayankhongor Province, Mongolia, in July 2017 (Table 1). The manufacturing processes of these dairy products were similar. All dairy samples were collected in sterile tubes with sample protector for RNA/DNA (TaKaRa Bio Inc., Shiga, Japan) for DNA extraction and into sterile tubes with $\mathrm{CaCO}_{3}$ and starch for bacterial isolation. The dairy samples were immediately placed in a portable sampling box with ice and transported to the laboratory. Dairy samples collected from Inner Mongolia were transported to our laboratory within $8 \mathrm{~h}$ and those from Bayankhongor Province within 1 to $2 \mathrm{~d}$. The samples were stored at $-80^{\circ} \mathrm{C}$ and analyzed as soon as possible. In this study, samples of KIM, KM, and KM that underwent PMA treatment were designated PKIM, PKM, and PCM, respectively.

\section{PMA Treatment}

The PMA treatment was performed as described by Erkus et al. (2016). Briefly, $1 \mathrm{~mL}$ of dairy sample was treated with $25 \mu \mathrm{L}$ of $2 \mathrm{mM}$ PMA (Biotium Inc., Fremont, CA), followed by thorough mixing and incubation in the dark for 5 min at room temperature, with additional mixing at 1-min intervals. Then, the samples were exposed to the PhAST Blue-PhotoActivation System (GenIUL, IUL S.A., Barcelona, Spain) on ice for 5 min. Cells were harvested by centrifugation at $6,800 \times$ $g$ for $5 \mathrm{~min}$ at $4^{\circ} \mathrm{C}$.

\section{DNA Extraction}

Genomic DNA was extracted from the samples using the Omega eZNA Soil DNA Kit (Qiagen, Hilden, Germany) according to the manufacturer's protocol. The quality of the DNA was checked by electrophoresis on a $0.8 \%$ agarose gel (Liuyi Biotechnology, Beijing, China) and spectrophotometry (optical density ratio at 260/280 nm; Thermo Fisher Scientific, Waltham, MA). The extracted DNA samples were stored at $-20^{\circ} \mathrm{C}$ until use.

\section{Amplification of Full-Length 16S rRNA and PacBio SMRT Sequencing}

Bacterial 16S rRNA was amplified using PCRBIO Taq DNA polymerase (PCR Biosystems Ltd., London, UK) and the 27F (5'-GAGAGTTTGATCCTGGCTCAG-3') and 1492R (5'-TACGGCTACCTTGTTACGACTT-3') primers. The primers contained a set of 16-nucleotide barcodes that were used to barcode SMRT sequencing of the full-length $16 \mathrm{~S}$ rRNA gene. The volume of the 


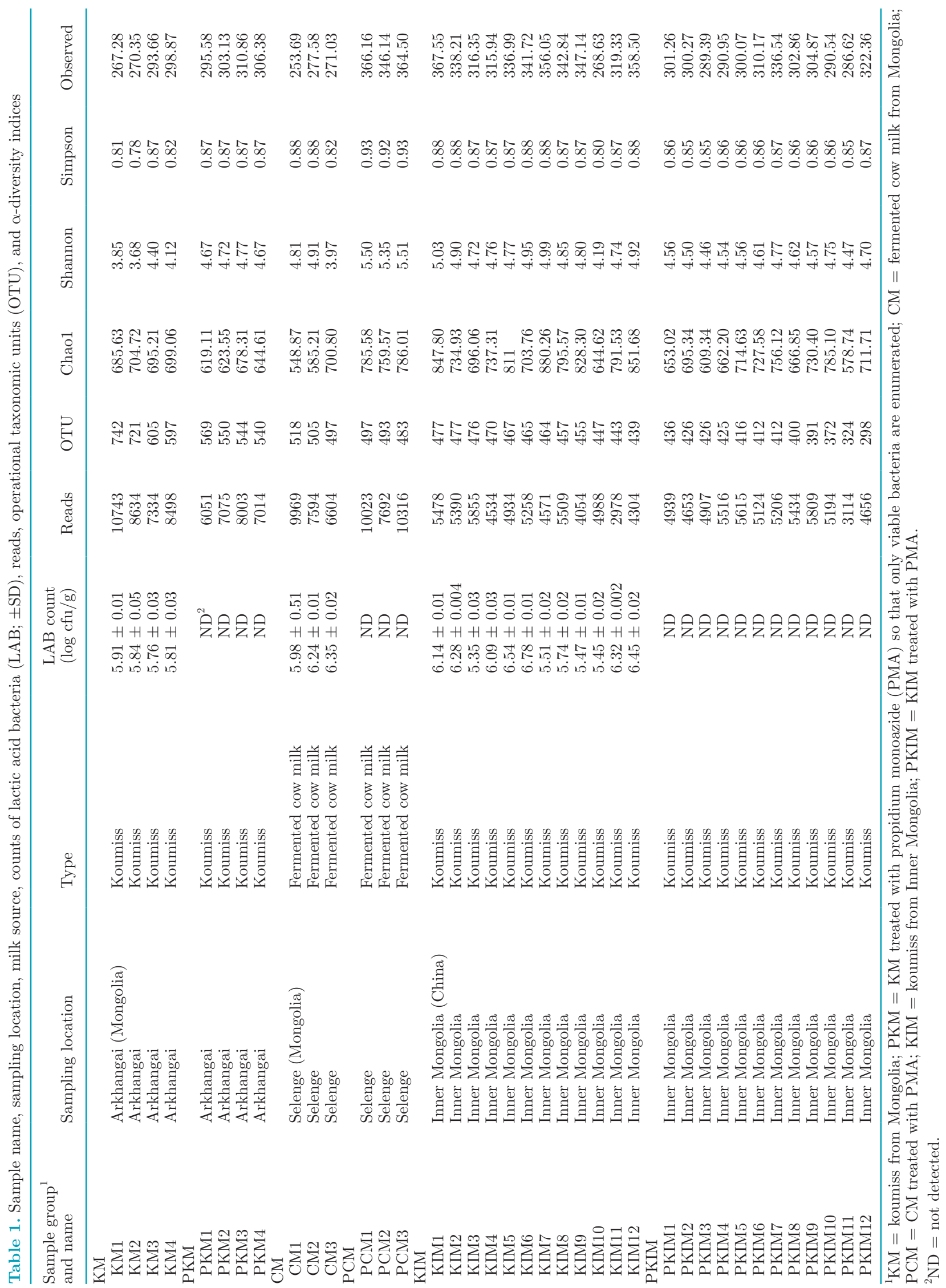


reaction mixture was $50 \mu \mathrm{L}$, comprising $10 \mu \mathrm{L}$ of $5 \times$ PCRBIO reaction buffer, $2 \mu \mathrm{L}$ of forward primer $(10$ $\mu M), 2 \mu \mathrm{L}$ of reverse primer $(10 \mu M), 1 \mu \mathrm{L}$ of template DNA, $1 \mu \mathrm{L}$ of PCRBIO Taq DNA polymerase (5 $\mathrm{U} / \mu \mathrm{L}$ ) (all from PCR Biosystems Ltd.) and $34 \mu \mathrm{L}$ of double-distilled $\mathrm{H}_{2} \mathrm{O}$. The PCR program was $95^{\circ} \mathrm{C}$ for $4 \mathrm{~min}$; followed by 30 cycles of $95^{\circ} \mathrm{C}$ for $60 \mathrm{~s}, 60^{\circ} \mathrm{C}$ for $45 \mathrm{~s}$, and $72^{\circ} \mathrm{C}$ for $60 \mathrm{~s}$; with a final extension at $72^{\circ} \mathrm{C}$ for 7 min (2720 Thermal Cycler, Applied Biosystems, Foster City, CA; Liu et al., 2015). The quality of PCR products was checked using an Agilent DNA 1000 Kit and an Agilent 2100 Bioanalyzer (Agilent Technologies, Santa Clara, CA) following the manufacturer's protocol. The amplicons were purified and used to construct DNA libraries using the Pacific Biosciences Template Prep Kit 2.0. The amplicons were sequenced using the P6-C4 chemistry on a PacBio RS II instrument (Pacific Biosciences). Quality control for PCR and sequence preprocessing was performed as described previously (Mosher et al., 2013).

\section{Sequence Processing and Bioinformatics Analysis}

Raw data were processed using the RS_ReadsOfinsert.1 protocol in the SMRT portal (ver. 2.7, Pacific Biosciences). Raw reads were filtered using the Quantitative Insights into Microbial Ecology (QIIME) package (ver. 1.7; Caporaso et al., 2010) according to the following criteria: (1) minimum full passes, up to 5; (2) minimum predicted accuracy, 90; (3) minimum read length of inserts, 1,400; and (4) maximum read length, 1,800 (Hou et al., 2015). The barcode and primer sequences were removed to create the data set. Next, PyNAST and UCLUST were applied to align the extracted high-quality sequences under $100 \%$ clustering of sequence identity to obtain representative sequences (Caporaso et al., 2010; Edgar, 2010). The representative sequences were clustered to obtain operational taxonomic units (OTU) with a $97 \%$ identity threshold using the UCLUST algorithm (Lozupone and Knight, 2005). The Ribosomal Database Project II database was used to assign the taxonomy of each OTU representative sequence at an $80 \%$ bootstrap threshold (Cole et al., 2007). For downstream analysis, a de novo taxonomic tree was constructed in FastTree using the representative OTU set (Price et al., 2009). The $\alpha$-diversity was evaluated by the Shannon-Wiener, Simpson's diversity, Chao1, and observed species index. Weighted and unweighted principal coordinate analyses (PCoA) based on UniFrac metrics were performed to assess the microbiota structure (Lozupone and Knight, 2005).

\section{Enumeration and Isolation of LAB}

The pour-plate method was applied to enumerate total LAB counts in the dairy samples (Yu et al., 2015). Briefly, $1 \mathrm{~g}$ of homogenized sample was aseptically diluted in $9 \mathrm{~mL}$ of sterile physiological saline $(0.85 \%$ wt/vol $\mathrm{NaCl}$ ) and thoroughly mixed by vortex. Following preparation of serial 10-fold dilutions, $1 \mathrm{~mL}$ of appropriate dilutions was mixed with molten de Man, Rogosa, and Sharpe (MRS; Difco Laboratories, Detroit, MI) agar supplemented with $0.01 \%$ (vol/vol) cycloheximide (Solarbio, Beijing, China). Sample dilutions (0.2 $\mathrm{mL}$ ) were also spread on MRS and M17 (Oxoid Ltd., Basingstoke, UK) agar. The plates were incubated at $30^{\circ} \mathrm{C}$ for $48 \mathrm{~h}$ under anaerobic conditions. Colonies with distinct morphologies (e.g., color, shape, and size) were randomly selected, streaked on the appropriate solid medium, and their Gram reactions and catalase production were analyzed. Skim milk containing $0.1 \%$ (wt/ vol) yeast powder was added to the purified isolates, which were stored at $-80^{\circ} \mathrm{C}$ until needed.

\section{Identification of $L A B$}

Total genomic DNA was extracted from the isolates using the cetyltrimethylammonium bromide (CTAB) method (Zhu et al., 1993). Next, $100 \mathrm{ng} / \mu \mathrm{L}$ purified DNA was used as the template for PCR amplification of the 16S rRNA gene using an automatic thermal cycler (PTC-200, MJ Research, Waltham, MA) and the primers 16S-FA (5'-AGAGTTTGATCCTGGCTCAG-3') and 16S-RA (5'-CTACGGCTACCTTGTTACGA-3') (Scarpellini et al., 2002). Each $50-\mu \mathrm{L}$ PCR contained $2 \mu \mathrm{L}$ of DNA template $(100 \mathrm{ng} / \mu \mathrm{L}), 5 \mu \mathrm{L}$ of $10 \times$ buffer $\left(\mathrm{Mg}^{2+}\right), 4 \mu \mathrm{L}$ of dNTPs $(10 \mathrm{mmol} / \mathrm{L}), 1.5 \mu \mathrm{L}$ of primer 16S-FA $(10 \mathrm{pmol} / \mu \mathrm{L}), 1.5 \mu \mathrm{L}$ of primer $16 \mathrm{~S}-\mathrm{RA}$ $(10 \mathrm{pmol} / \mu \mathrm{L}), 0.5 \mu \mathrm{L}$ of Taq DNA polymerase $(5 \mathrm{U} /$ $\mu \mathrm{L}$, TaKaRa Bio Inc.), and $35.5 \mu \mathrm{L}$ of triple-distilled water. The PCR was conducted as follows: $94^{\circ} \mathrm{C}$ for $5 \mathrm{~min}$; followed by 30 cycles of $94^{\circ} \mathrm{C}$ for $1 \mathrm{~min}, 58^{\circ} \mathrm{C}$ for $1 \mathrm{~min}$, and $72^{\circ} \mathrm{C}$ for $2 \mathrm{~min}$; followed by $72^{\circ} \mathrm{C}$ for 10 min, and holding at $4^{\circ} \mathrm{C}$ (Yu et al., 2012). The purified products were sequenced by Majorbio Bio-Pharm Technology Corp. (Shanghai, China). The sequences were assembled using SeqMan in DNAStar software and identified using the basic local alignment search tool (BLAST; https://blast.ncbi.nlm.nih.gov/Blast .cgi) based on taxonomic assignment with the closest relative (99\% similarity level cut-off; Altschul et al., 1997). Then, MEGA ver. 6.0 software (http://www .megasoftware.net) was used to construct phylogenetic trees using the neighbor-joining (NJ) method. 


\section{Nucleotide Sequence Accession Numbers}

All of the PacBio SMRT sequencing data reported in this study were deposited at Metagenomic Rapid Annotations using Subsystems Technology (MG-RAST) database (http://metagenomics.anl.gov/; accession no. mgp87678). All LAB isolate sequences were deposited in the National Center for Biotechnology Information database (https://www.ncbi.nlm.nih.gov/) under the accession numbers MG755331 to MG755377 and MK144560 to MK144614.

\section{Statistical Analysis}

The Kruskal-Wallis test was used to evaluate differences in bacterial population and $\alpha$-diversity between sample groups. Multivariate ANOVA (MANOVA) of UniFrac distance was analyzed in Matlab (The MathWorks, Natick, MA). Linear discriminant analysis (LDA) effect size method based on a normalized relative abundance matrix was to identify the significant differences between cheese samples. The $\mathrm{R}$ package, version 3.1.2 (https://www.r-project.org/) and Origin software (version 8.5, OriginLab Corporation, Hampton, MA) were used to generate the graphs.

\section{RESULTS}

\section{Sequence Abundance and Diversity}

In total, 233,570 high-quality sequencing reads were generated from 38 samples of homemade dairy products (average, 6,146.58; range, 2,978-10,743; $\mathrm{SD}, 1,918)$. After sequence alignment and clustering, 18,136 unique and representative OTU were obtained (average, 477.26; range, 298-742; SD, 88). The Shannon index (range, 3.68-5.51), Chao1 index (range, 548.87-880.26), Simpson index (range, 0.78-0.93), and number of species (range, 253.69-367.55; Table 1) indicated that the dairy products had a high level of bacterial diversity. Furthermore, the Shannon diversity and rarefaction curves indicated that most of the bacterial diversity was captured at the current sequencing depth (Supplemental Figure S1; https://doi.org/10.3168/jds .2018-15756).

\section{Bacterial Community Composition}

Using Greengenes (ver. 13.8; http://greengenes.lbl .gov/) and the Ribosomal DatabaseProject (RDP) II database (ver. 11.4; http://rdp.cme.msu.edu/), a total of 13 phyla were identified. The phyla (mean relative abundance $>1 \%$ ) Firmicutes $(77.22 \%$ ) and Proteobac- teria $(22.70 \%)$ predominated but varied in abundance (Supplemental Table S1; https://doi.org/10.3168/jds .2018-15756). Firmicutes was the most prevalent phylum in the KM, KIM, CM, and PKIM samples, whereas Proteobacteria was predominant in the PKM and PCM samples.

More than 121 bacterial genera were identified. Of these, Lactobacillus (Lb.; 40.84\%), Streptococcus $(34.13 \%)$, Enterobacter (17.99\%), Serratia (2.68\%), and Lactococcus ( $\boldsymbol{L}$ c.; $1.47 \%$ ) had an average relative abundance of $>1 \%$ (Figure 1A). Pseudomonas and Serratia were identified in KIM samples, constituting 1.58 and $3.77 \%$, respectively, and were at lower abundance in the Mongolian samples. Among the 195 bacterial species detected, Streptococcus parauberis (33.77\%), Lb. helveticus (32.96\%), Enterobacter xiangfangensis (13.70\%), Enterobacter hormaechei (1.86\%), and Lc. lactis $(1.22 \%)$ predominated. In particular, in the untreated sample groups, bacterial composition at the species level varied among the types of dairy products (Figure 1): Lb. helveticus (76.86\%), Strep. parauberis (62.65\%), and Lb. delbrueckii $(56.69 \%)$ were the most abundant species in the KM, KIM, and CM samples, respectively. Lactobacillus helveticus was considered the second most dominant strain in KIM, at 26.85\%. Furthermore, the relative abundance of Lc. lactis detected in KM was higher than that in KIM and CM (Figure 1B; Supplemental Table S2; https://doi.org/10.3168/ jds.2018-15756).

\section{Effect of PMA Treatment on Bacterial Community Composition}

The relative abundance of Firmicutes decreased from 81.74 to $1.09 \%$ and that of Proteobacteria increased from 18.25 to $98.73 \%$ between KM and PKM samples (Supplemental Table S1; https://doi.org/10.3168/jds .2018-15756). The relative abundances of these phyla in the CM and PCM samples exhibited similar trends, possibly because these samples were from the same collection batch. However, the relative abundances at the phylum level differed among samples from Inner Mongolia. At the genus level, Enterobacter predominated in both the PKM (>97\%) and PCM (>64\%) samples but varied in abundance. The abundance of Lactobacillus in the PKIM samples tended to increase after PMA treatment (Figure 1A). The predominant species in the PKM, PKIM, and PCM samples were E. xiangfangensis $(74.22 \%)$, Lb. helveticus $(45.26 \%)$, and $E$. xiangfangensis $(49.73 \%)$, respectively, compared with Lb. helveticus (76.86\%), Strep. parauberis (62.65\%), and $L b$. delbrueckii $(56.79 \%)$ in the KM, KIM, and CM samples, respectively (Figure 1B). Lactococcus lactis 

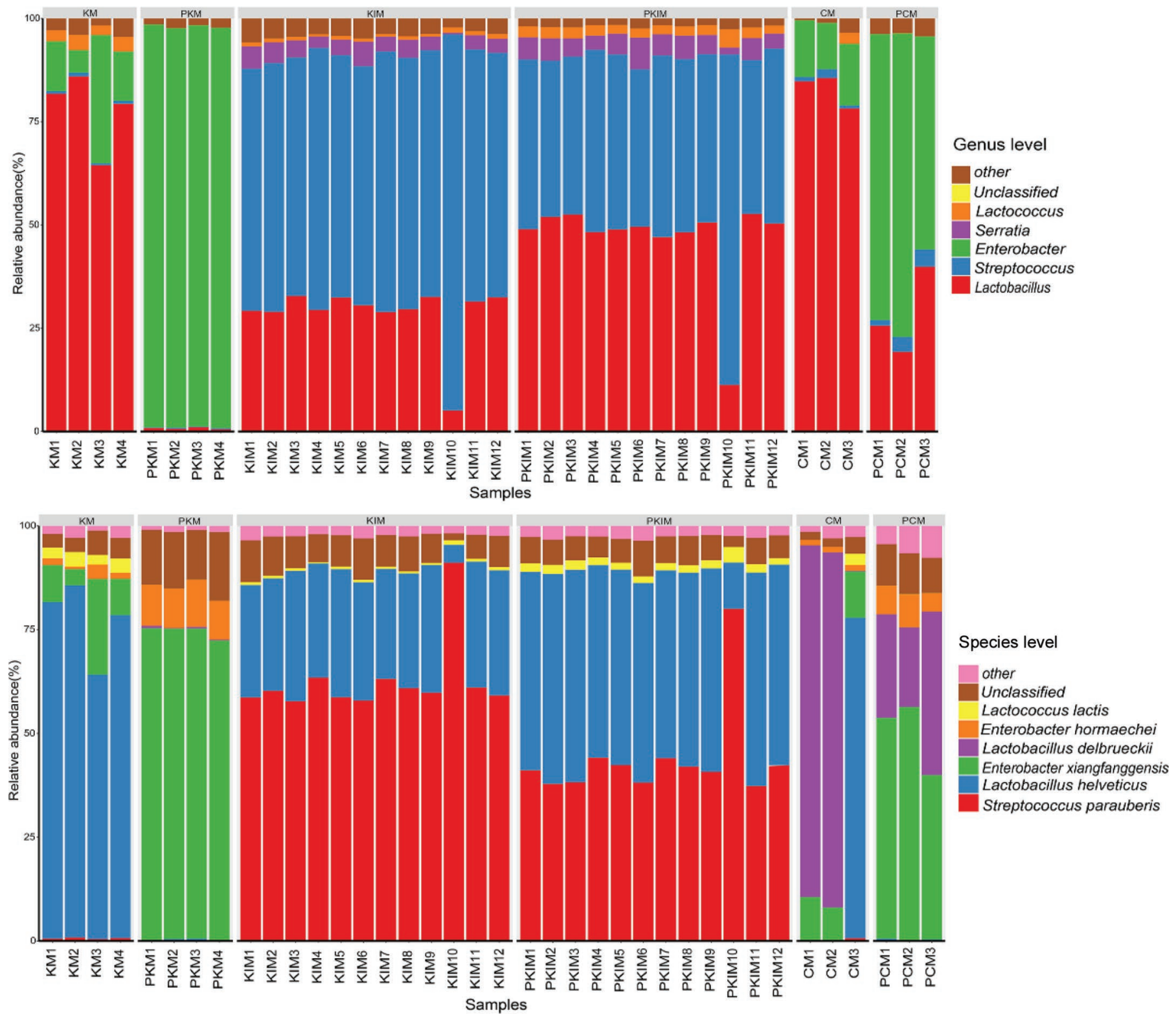

Figure 1. Relative abundance of bacterial composition in traditional fermented dairy foods samples at the genus level (top) and the species level (bottom). $\mathrm{KM}=$ koumiss from Mongolia; PKM = KM treated with propidium monoazide (PMA) so that only viable bacteria are enumerated; $\mathrm{KIM}=$ koumiss from Inner Mongolia; PKIM = KIM treated with PMA; CM = fermented cow milk from Mongolia; PCM $=$ CM treated with PMA.

was detected in the KM and PKM samples at relative abundances of 2.95 and $<0.02 \%$, respectively (Figure 1B; Supplemental Table S2; https://doi.org/10.3168/ jds.2018-15756). The abundance of E. hormaechei was significantly higher in the PKM and PCM samples than in the KM and CM samples. Anoxybacillus flavithermus was detected in the PCM samples at a relative abundance of $>1 \%$. The relative abundances of $L b$. kefiri and $L b$. hamsteri were significantly higher in the KIM samples than in the PKIM samples $(P<0.01)$, and the relative abundances of $L b$. kefiri, Lb. gallinarum, $L b$. helveticus, Lb. kefiri, Lc. raffinolactis, Leuconostoc citreum, and Leuconostoc mesenteroides were significantly higher in the KM samples than in the PKM samples $(P<0.01)$.

\section{Bacterial Profiles of the Dairy Products}

A PCoA based on the weighted (principal components 1 and 3 accounted for 90.53 and $1.62 \%$ of the 


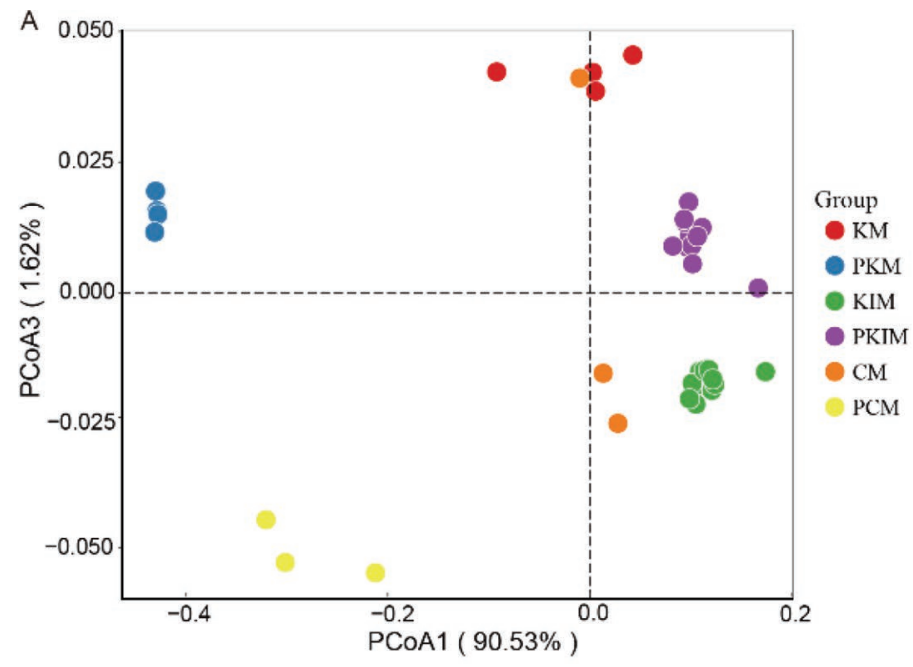

Weighted PCOA



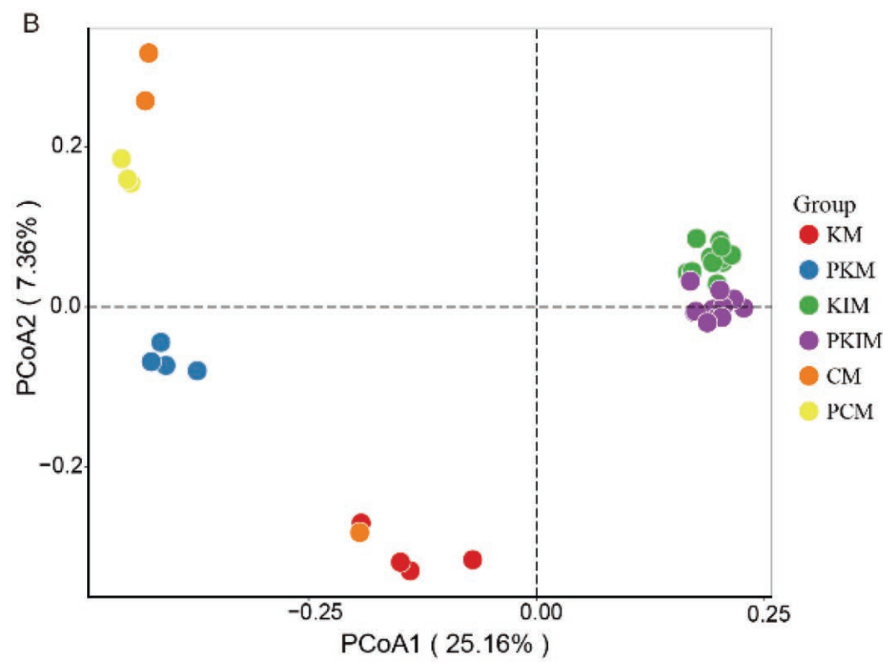

Unweighted PCOA

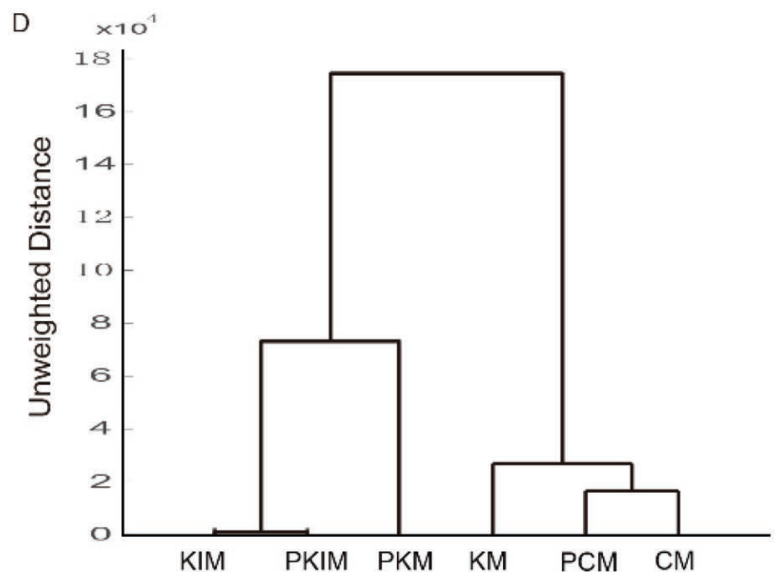

Figure 2. UniFrac weighted (A) and unweighted (B) principal coordinate analysis (PCoA) scores plot based on principal components 1, 2, and 3. Clustering of dairy sample groups based on (C) weighted and (D) unweighted UniFrac distances calculated using multivariate ANOVA. $\mathrm{KM}=$ koumiss from Mongolia; PKM = KM treated with propidium monoazide (PMA) so that only viable bacteria are enumerated; KIM = koumiss from Inner Mongolia; PKIM = KIM treated with PMA; CM = fermented cow milk from Mongolia; PCM = CM treated with PMA.

total variance, respectively) and unweighted (principal components 1 and 2 accounted for 25.16 and $7.36 \%$ of the total variance, respectively) UniFrac distances showed that the bacterial community composition differed among the types of dairy samples (Figure 2). Both weighted and unweighted PCoA score plots displayed a clear clustering pattern with good separation; the only overlap was that between one fermented cow milk sample and the koumiss samples from Mongolia. The differences in bacterial community composition were confirmed by MANOVA.

The $\alpha$-diversity indices differed significantly among the dairy sample groups $(P=0.00018$ to 0.0015 ; Figure $3)$. The KIM samples had the highest Chao 1 index value, suggesting a high level of bacterial richness, whereas PCM had the highest Shannon and Simpson indices, indicating a high level of bacterial diversity. The $\alpha$-diversity indices of the KIM and PKIM samples differed significantly $(P<0.01)$, as did the Chao 1 , Shannon, and Simpson indices between the KM and PKM samples $(P<0.05$; Figure 3$)$. Therefore, PMA treatment before DNA extraction affected the bacterial diversity of the koumiss samples. The $\alpha$-diversity values also differed significantly between the KM and KIM samples.

Linear discriminant analysis of the effect size resulted in the identification of 31 bacterial clades with significantly different relative abundances: 15 in KM, 7 in KIM, 5 in PKIM, 2 in PKM, and 2 in CM samples. The relative abundances of $L b$. kefiranofaciens in PKIM; 

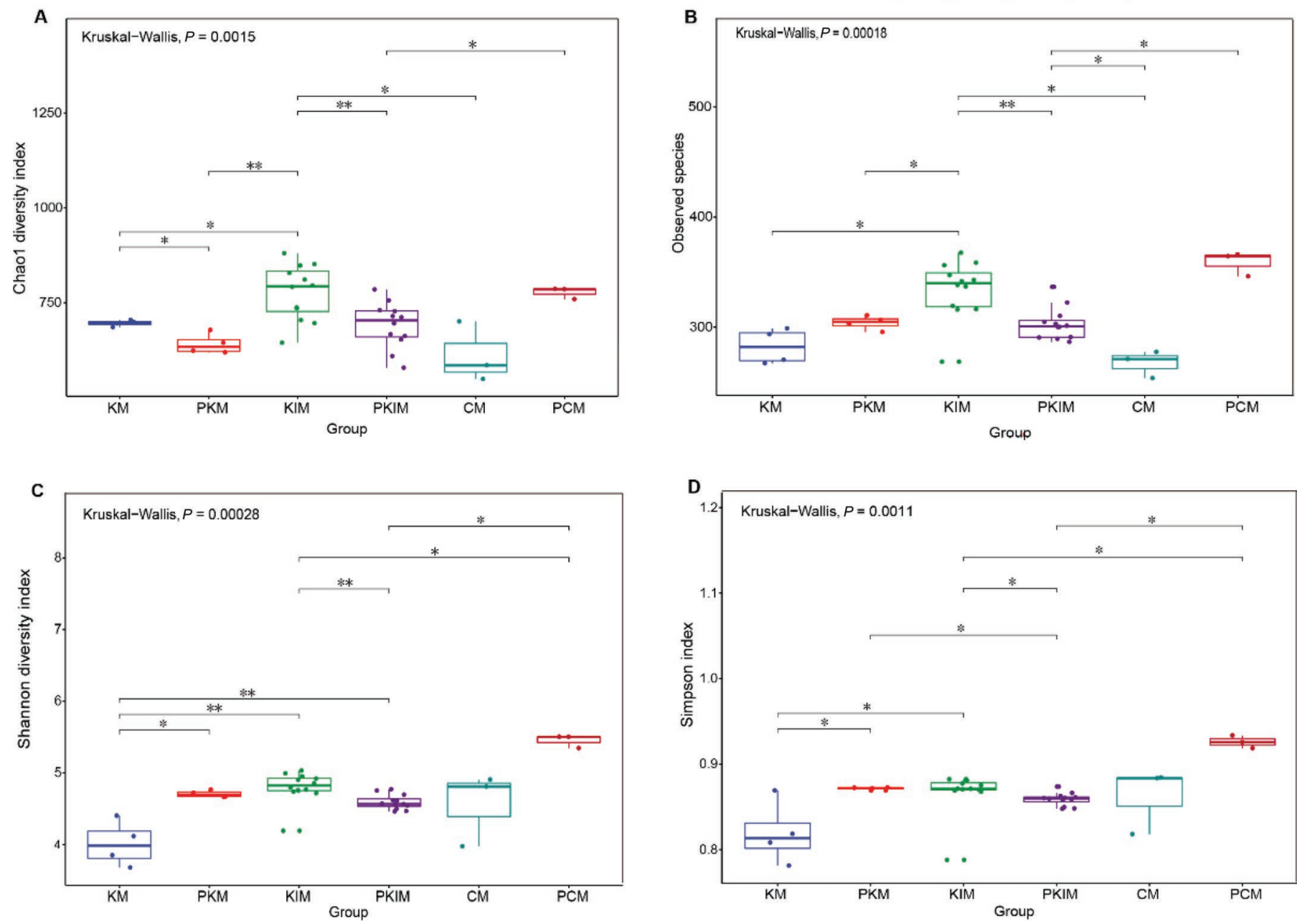

Figure 3. Boxplots of $\alpha$-diversity indexes. Correlation between all $\alpha$-diversity indices of traditional fermented dairy foods samples with propidium monoazide (PMA) and non-PMA treatments. (A) Chao 1, (B) observed species, (C) Shannon index, and (D) Simpson index. KM = koumiss from Mongolia; PKM = KM treated with propidium monoazide (PMA) so that only viable bacteria are enumerated; KIM = koumiss from Inner Mongolia; PKIM = KIM treated with PMA; CM = fermented cow milk from Mongolia; PCM = CM treated with PMA. Filled dots represent each sample. ${ }^{*} P<0.05 ; * * P<0.01$.

Strep. parauberis in KIM; Lb. helveticus, Lb. kefiri, and Lc. lactis in KIM; and Lactobacillus in the CM samples were significantly higher than in the other dairy products, in agreement with the relative abundances at the genus and species levels (Figure 4). No species was identified at higher abundance than any other in the PCM samples.

\section{Enumeration and Identification of $L A B$}

The viable LAB counts of the 19 samples ranged from $5.45 \pm 0.02$ to $6.78 \pm 0.01 \log \mathrm{cfu} / \mathrm{mL}$ (Table 1 ). In total, 102 pure LAB isolates were obtained from the dairy samples. The 102 isolates were identified as $L b$. helveticus (70 strains), Lb. kefiranofaciens (11 strains),
Strep. thermophilus (8 strains), Lb. delbrueckii ssp. bulgaricus (5 strains), Lb. fermentum (5 strains), $L b$. buchneri (1 strain), Lb. plantarum (1 strain), and $L b$. paracasei (1 strain).

\section{DISCUSSION}

We investigated the bacterial community composition of PMA-treated and untreated traditional Mongolian dairy products using PacBio SMRT technology and full-length $16 \mathrm{~S}$ rRNA gene sequences. Both pure culture and PacBio SMRT technology showed that $L b$. helveticus was the most abundant species, which is in accordance with a previous study (Sun et al., 2014). However, several species typically present in dairy 



Figure 4. Identification of discriminant taxa between traditional fermented dairy foods by linear discriminant analysis (LDA) of the effect size. (A) Horizontal bar chart showing significant discriminant taxa in samples. KIM = koumiss from Inner Mongolia; KM = koumiss from Mongolia; PKIM = KIM treated with propidium monoazide (PMA) so that only viable bacteria are enumerated; PKM $=$ KM treated with PMA; CM = fermented cow milk from Mongolia, represented by red, green, blue, purple, and cyan, respectively. (B) Cladogram of the microbiota. Significant discriminant taxon nodes of the KIM, KM, PKIM, PKM, and CM are represented. Branch areas are shaded according to the highest-ranked variety for that taxon. The LDA score indicates the level of differentiation among dairy samples among different groups. A threshold value of 3.5 was used as the cut-off.

foods; for example, Lb. sakei, Lc. lactis, Lc. raffinolactis, and Leu. mesenteroides were not detected by culture, which may due to the small number of isolates per samples. It is also possible that the bacterial content of the samples was too low and insufficient for detection. In addition, E. xiangfangensis and E. hormaechei were excluded due to negative Gram staining when selecting the LAB isolates by the pure culture method. However, pure culture methods are necessary for isolating pure strains, for research on probiotics, and for industrial applications.

In this study, 195 bacterial species of 121 genera and 13 phyla were identified in samples not treated with PMA. Gesudu et al. (2016) identified 148 bacterial species in 82 genera and 8 phyla in koumiss by PacBio SMRT sequencing. Lactobacillus and Streptococcus were the predominant genera in untreated dairy samples.
Using conventional culture and molecular biological methods, Watanabe et al. (2008) found that Lactobacillus and Streptococcus predominated in Mongolian tarag. Most Lactobacillus species were detected in both KM and CM in the current study, as also reported by Sun et al. (2014); this is likely due to their greater acid tolerance (An et al., 2015). In this study, Lb. delbrueckii predominated in $\mathrm{CM}$, in accordance with previous reports (Sun et al., 2014; Liu et al., 2016). Lactobacillus helveticus and Strep. parauberis predominated in the KM and KIM samples, respectively. Lactobacillus acidophilus, Lb. helveticus, Lb. fermentum, and $L b$. plantarum, but not Strep. parauberis, are typically the predominant LAB strains in koumiss (Oki et al., 2014; An et al., 2015; Shangpliang et al., 2018). Lactococcus lactis and Lc. garvieae represented a small proportion of the bacterial community in koumiss. 
The bacterial community compositions of traditional fermented dairy products from Mongolia and Inner Mongolia may vary according to sampling location, type of raw milk, and other factors. The dominant bacteria in koumiss in our study differed from those in previous reports (Hao et al., 2010; Oki et al., 2014). Differences in location and climate can affect the bacterial community composition of the product, as can the type of raw milk used (Liu et al., 2015). In this study, E. xiangfangensis and Lb. delbrueckii predominated in KM and CM, respectively. Similarly, Watanabe et al. (2008) reported that differences among the animal species from which the milk was sourced influenced the bacterial diversity of traditional fermented milks.

The PMA treatment caused significant changes in the predominant species in the dairy food samples analyzed. The relative abundances of E. xiangfangensis and E. hormaechei were significantly higher in PKM and PCM than in KM and CM, respectively. Enterobacter xiangfangensis was first isolated from traditional sourdough in Heilongjiang Province, China, and was classified into the Enterobacter cloacae complex of the Enterobacteriaceae (Gu et al., 2014). We assumed that the raw milk contained a great number of E. xiangfangensis, and that this community had stronger survivability during fermentation processing. Its resistance to PMA treatment may be due to its gram-negative nature (Tantikachornkiat et al., 2016). Using denaturing gradient gel electrophoresis, Ringo et al. (2014) found one unique band indicating Enterobacter species. Streptococcus parauberis is a gram-positive, catalasenegative species frequently isolated from the milk of cows with IMI (Williams and Collins, 1990; Varhimo et al., 2007). Its relative abundance was decreased by PMA treatment, whereas that of $L b$. helveticus increased. The raw milk likely contained a large number of Strep. parauberis, a number of which were killed by the acidity generated during fermentation. An et al. (2015) reported that Strep. parauberis made up $16 \%$ of the LAB community of fresh mare milk from Inner Mongolia, and that Lactobacillus spp. predominated $(81 \%)$ in chigee (a traditional fermented mare milk).

Streptococcus parauberis, Anoxybacillus flavithermus, Enterobacter spp., and other opportunistic pathogens were detected in the dairy samples. Streptococcus parauberis in bovine milk is related to mastitis and is frequently detected on the lips, skin, and udders of cows, as well as in raw milk from Inner Mongolia (Williams and Collins, 1990; An et al., 2015). It is worth noting that the relative abundance of Anoxybacillus flavithermus, E. xiangfangensis, and E. hormaechei increased among PMA-treated samples, demonstrating that those species may be viable. Our results suggest that traditional Mongolian dairy products pose a health risk, likely because of the hygiene of the production methods used. Unpasteurized fresh mare and cow milks are fermented using natural starters, increasing the potential for contamination by microorganisms; indeed, the starters may be present in fresh milk. Raw milk may become contaminated due to inadequate hygiene during premilking, of milk handlers and utensils, and during milking and storage (Afif et al., 2008; Garedew et al., 2012). Thus, appropriate hygiene measures are required to improve the quality of milk and prevent outbreaks of food-borne infectious diseases.

We detected apparent differences in microbiota communities between PMA-treated and untreated sample groups, as revealed by PCoA, MANOVA, and $\alpha$-diversity indices, suggesting that PMA permitted an accurate assessment of the bacterial community structure. The PCM sample had the highest Shannon and Simpson indices, which may be a result of the large proportions of some species. At the current sequencing depth, sequences of low-abundance species may have been covered up by high-abundance species and thus could not be detected. Low-abundance microbes were detected more readily when partial nonviable bacteria in high-abundance bacteria were screened by PMA treatment. Compared with the CM sample group, about 24 species with relative abundance $<0.01 \%$ were detected in the PCM sample group, such as Strep. thermophilus, Lb. mucosae, Lb. gasseri, and Bacillus cereus (Supplemental Table S2; https://doi.org/10.3168/jds .2018-15756). Microbial community diversity is affected by sequencing depth and PCR amplification procedure (Huber et al., 2007; Kuczynski et al., 2010). In the case of low sequencing depth, the reflected community structure is mostly composed of high-abundance microbes. In addition, PCR amplification is not capable of proportional amplification, resulting in amplification of the high-abundance microorganisms during PCR and reducing the probability of rare microbes being discovered (Pedrós-Alió, 2006). The production, collection, storage, and transportation of dairy products affects microbial diversity and dairy quality. Enumeration results of viable LAB (ranging from 5.45 to $6.78 \mathrm{log} \mathrm{cfu} /$ $\mathrm{mL}$ ) suggested low viability of LAB in the Mongolian dairy samples. Meanwhile, in this study, long-term transportation might have caused loss of viable bacteria, thus affecting the microbial community structure after PMA treatment.

The PMA treatment had a significant effect on the relative abundances of some species. Determining bacterial population dynamics in dairy foods is important because the balance of viable and nonviable bacteria has an important effect on final flavor and texture (Crow et al., 1995). The PMA treatment has been used to identify viable and nonviable LAB strains in dairy 
foods; for example, Lb. paracasei (Scariot et al., 2018), Lb. bulgaricus (Shao et al., 2016), Strep. thermophilus, and Lb. acidophilus (Garcia-Cayuela et al., 2009). Despite the benefits of comprehensive microbial profiling based on total DNA from samples, the bacterial population structure may be muddled by amplifying DNA from injured or dead bacteria. Propidium monoazide treatment coupled with PacBio SMRT sequencing is a powerful approach for bacterial diversity profiling within dairy samples. Using PMA as a precursor to PacBio SMRT sequencing has significant advantages in that it allows easy, quick, and more accurate analysis. Our results suggest that PMA combined with PacBio SMRT sequencing based on full-length bacterial $16 \mathrm{~S}$ rRNA gene permits an accurate assessment of bacterial population structure in traditional fermented dairy foods.

\section{CONCLUSIONS}

We investigated the bacterial communities of traditional fermented dairy foods from Mongolia and Inner Mongolia using PMA and PacBio SMRT sequencing; we isolated $102 \mathrm{LAB}$ strains to obtain a more complete overview on bacterial community structure. Full-length $16 \mathrm{~S}$ rRNA sequencing identified 195 bacterial species in 121 genera and 13 phyla in PMA-treated and untreated dairy samples. The PMA treatment induced significant changes in bacterial community structure, suggesting its utility for selective analysis of viable bacteria in dairy foods. Thus, PMA treatment and PacBio SMRT sequencing enable monitoring of the dynamics of bacteria communities during dairy fermentation.

\section{ACKNOWLEDGMENTS}

This research was supported by the China Agriculture Research System (Beijing, China; Grant CARS-36 to HZ) and Key Project of the Inner Mongolia Science and Technology Plan (Inner Mongolia, China; Grant No. 201603001).

\section{REFERENCES}

Afif, A., M. Faid, F. Chigr, and M. Najimi. 2008. Survey of the microbiological quality of the raw cow milk in the Tadla area of Morocco. Int. J. Dairy Technol. 61:340-346.

Altschul, S. F., T. L. Madden, A. A. Schaffer, J. Zhang, Z. Zhang, W. Miller, and D. J. Lipman. 1997. Gapped BLAST and PSI-BLAST: A new generation of protein database search programs. Nucleic Acids Res. 25:3389-3402.

Amir, A., A. Zeisel, O. Zuk, M. Elgart, S. Stern, O. Shamir, P. J. Turnbaugh, Y. Soen, and N. Shental. 2013. High-resolution microbial community reconstruction by integrating short reads from multiple 16S rRNA regions. Nucleic Acids Res. 41:e205.
An, Y., Y. Adachi, and Y. Ogawa. 2015. Classification of lactic acid bacteria isolated from chigee and mare milk collected in Inner Mongolia. Anim. Sci. J. 75:245-252.

Cai, L., L. Ye, A. H. Y. Tong, S. Lok, and T. Zhang. 2013. Biased diversity metrics revealed by bacterial $16 \mathrm{~S}$ pyrotags derived from different primer sets. PLoS One 8:e53649.

Caporaso, J. G., K. Bittinger, F. D. Bushman, T. Z. DeSantis, G. L. Andersen, and R. Knight. 2010. PyNAST: A flexible tool for aligning sequences to a template alignment. Bioinformatics 26:266-267.

Cole, J. R., B. Chai, R. J. Farris, Q. Wang, A. S. Kulamsyedmohideen, D. M. Mcgarrell, A. M. Bandela, E. Cardenas, G. M. Garrity, and J. M. Tiedje. 2007. The ribosomal database project (RDPII): Introducing myRDP space and quality controlled public data. Nucleic Acids Res. 35:D169-172.

Crow, V. L., F. G. Martley, T. Coolbear, and S. J. Roundhill. 1995 The influence of phage-assisted lysis of Lactococcus lactis ssp. lactis ML8 on cheddar cheese ripening. Int. Dairy J. 5:451-472.

Edgar, R. C. 2010. Search and clustering orders of magnitude faster than BLAST. Bioinformatics 26:2460-2461.

Erkus, O., V. C. L. de Jager, R. T. C. M. Geene, I. van Alen-Boerrigter, L. Hazelwood, S. A. F. T. van Hijum, M. Kleerebezem, and E. J. Smid. 2016. Use of propidium monoazide for selective profiling of viable microbial cells during Gouda cheese ripening. Int. J. Food Microbiol. 228:1-9.

Galat, A., J. Dufresne, J. Combrisson, J. Thepaut, L. BoumgharBourtchai, M. Boyer, and C. Fourmestraux. 2016. Novel method based on chromogenic media for discrimination and selective enumeration of lactic acid bacteria in fermented milk products. Food Microbiol. 55:86-94.

Garcia-Cayuela, T., R. Tabasco, C. Pelaez, and T. Requena. 2009. Simultaneous detection and enumeration of viable lactic acid bacteria and bifidobacteria in fermented milk by using propidium monoazide and real-time PCR. Int. Dairy J. 19:405-409.

Garedew, L., A. Berhanu, D. Mengesha, and G. Tsegay. 2012. Identification of gram-negative bacteria from critical control points of raw and pasteurized cow milk consumed at Gondar town and its suburbs, Ethiopia. BMC Public Health 12:950.

Gesudu, Q., Y. Zheng, X. Xi, Q. Hou, H. Xu, W. Huang, H. Zhang, B. Menghe, and W. Liu. 2016. Investigating bacterial population structure and dynamics in traditional koumiss from Inner Mongolia using single molecule real-time sequencing. J. Dairy Sci. 99:7852-7863.

Gu, C., C. Li, L. Yang, and G. Huo. 2014. Enterobacter xiangfangensis sp. nov. isolated from Chinese traditional sourdough, and reclassification of Enterobacter sacchari Zhu et al. 2013 as Kosakonia sacchari comb. nov. Int. J. Syst. Evol. Microbiol. 64(Pt. 8):2650. https://doi.org/10.1099/ijs.0.064709-0.

Hao, Y., L. Zhao, H. Zhang, Z. Zhai, Y. Huang, X. Liu, and L. Zhang. 2010. Identification of the bacterial biodiversity in koumiss by denaturing gradient gel electrophoresis and species-specific polymerase chain reaction. J. Dairy Sci. 93:1926-1933.

Hou, Q., H. Xu, Y. Zheng, X. Xi, L. Kwok, Z. Sun, H. Zhang, and W. Zhang. 2015. Evaluation of bacterial contamination in raw milk ultra-high temperature milk and infant formula using single molecule, real-time sequencing technology. J. Dairy Sci. 98:8464-8472.

Huber, J. A., D. Mark Welch, H. G. Morrison, S. M. Huse, P. R. Neal, D. A. Butterfield, and M. L. Sogin. 2007. Microbial population structures in the deep marine biosphere. Science 318:97-100.

Jin, H., L. Mo, L. Pan, Q. Hou, C. Li, I. Darima, and J. Yu. 2018 Using PacBio sequencing to investigate the bacterial microbiota of traditional Buryatian cottage cheese and comparison with Italian and Kazakhstan artisanal cheeses. J. Dairy Sci. 101:6885-6896.

Klindworth, A., E. Pruesse, T. Schweer, J. Peplies, C. Quast, M. Horn, and F. O. Glockner. 2013. Evaluation of general 16S ribosomal RNA gene PCR primers for classical and next-generation sequencing-based diversity studies. Nucleic Acids Res. 41:e1.

Kuczynski, J., Z. Z. Liu, C. Lozupone, D. McDonald, N. Fierer, and R. Knight. 2010. Microbial community resemblance methods differ in their ability to detect biologically relevant patterns. Nat. Methods $7: 813-819$ 
Li, J., Y. Zheng, H. Xu, X. Xi, Q. Hou, S. Feng, L. Wuri, Y. Bian, Z. Yu, L. Y. Kwok, Z. Sun, and T. Sun. 2017. Bacterial microbiota of Kazakhstan cheese revealed by single molecule real time (SMRT) sequencing and its comparison with Belgian, Kalmykian and Italian artisanal cheeses. BMC Microbiol. 17:13.

Liu, W., J. Yu, Z. Sun, Y. Song, X. Wang, H. Wang, T. Wuren, M. Zha, B. Menghe, and H. Zhang. 2016. Relationships between functional genes in Lactobacillus delbrueckii ssp. bulgaricus isolates and phenotypic characteristics associated with fermentation time and flavor production in yogurt elucidated using multilocus sequence typing. J. Dairy Sci. 99:89-103.

Liu, W., Y. Zheng, L. Y. Kwok, Z. Sun, J. Zhang, Z. Guo, Q. Hou, B. Menghe, and H. Zhang. 2015. High-throughput sequencing for the detection of the bacterial and fungal diversity in Mongolian naturally fermented cow's milk in Russia. BMC Microbiol. 15:45.

Lozupone, C., and R. Knight. 2005. UniFrac: A new phylogenetic method for comparing microbial communities. Appl. Environ. Microbiol. 71:8228-8235.

Mosher, J. J., E. L. Bernberg, O. Shevchenko, J. Kan, and L. A. Kaplan. 2013. Efficacy of a 3rd generation high-throughput sequencing platform for analyses of $16 \mathrm{~S}$ rRNA genes from environmental samples. J. Microbiol. Methods 95:175-181.

Nocker, A., C. Y. Cheung, and A. K. Camper. 2006. Comparison of propidium monoazide with ethidium monoazide for differentiation of live vs. dead bacteria by selective removal of DNA from dead cells. J. Microbiol. Methods 67:310-320.

Nocker, A., T. Richter-Heitmann, R. Montijn, F. Schuren, and R. Kort. 2010. Discrimination between live and dead cells in bacterial communities from environmental water samples analyzed by 454 pyrosequencing. Int. Microbiol. 13:59-65.

Oki, K., J. Dugersuren, S. Demberel, and K. Watanabe. 2014. Pyrosequencing analysis of the microbial diversity of airag, khoormog and tarag, traditional fermented dairy products of Mongolia. Biosci. Microbiota Food Health 33:53-64.

Oliver, J. D. 2005. The viable but nonculturable state in bacteria. J. Microbiol. 43:93-100.

Pedrós-Alió, C. 2006. Marine microbial diversity: Can it be determined? Trends Microbiol. 14:257-263.

Price, M. N., P. S. Dehal, and A. P. Arkin. 2009. FastTree: Computing large minimum evolution trees with profiles instead of a distance matrix. Mol. Biol. Evol. 26:1641-1650.

Ringo, E., R. Andersen, S. Sperstad, Z. G. Zhou, P. Ren, E. M. Breines, E. Hareide, G. J. Yttergard, K. Opsal, H. M. Johansen, A. K. Andreassen, A. Kousha, J. Godfroid, and W. Holzapfel. 2014. Bacterial community of koumiss from Mongolia investigated by culture and culture-independent methods. Food Biotechnol. 28:333-353.

Scariot, M. C., G. L. Venturelli, E. S. Prudencio, and A. C. M. Arisi. 2018. Quantification of Lactobacillus paracasei viable cells in probiotic yoghurt by propidium monoazide combined with quantitative PCR. Int. J. Food Microbiol. 264:1-7.

Scarpellini, M., D. Mora, S. Colombo, and L. Franzetti. 2002. Development of genus/species-specific PCR analysis for identification of Carnobacterium strains. Curr. Microbiol. 45:24-29.
Shangpliang, H. N. J., R. Rai, S. Keisam, K. Jeyaram, and J. P. Tamang. 2018. Bacterial community in naturally fermented milk products of Arunachal Pradesh and Sikkim of India analysed by high-throughput amplicon sequencing. Sci. Rep. 8:1532.

Shao, Y., Z. Wang, Q. Bao, and H. Zhang. 2016. Application of propidium monoazide quantitative real-time PCR to quantify the viability of Lactobacillus delbrueckii ssp. bulgaricus. J. Dairy Sci. 99:9570-9580.

Singer, E., B. Bushnell, D. Coleman-Derr, B. Bowman, R. M. Bowers, A. Levy, E. A. Gies, J. F. Cheng, A. Copeland, H. P. Klenk, S. J. Hallam, P. Hugenholtz, S. G. Tringe, and T. Woyke. 2016. Highresolution phylogenetic microbial community profiling. ISME J. 10:2020-2032.

Sun, Z., W. Liu, Q. Bao, J. Zhang, Q. Hou, L. Y. Kwok, T. Sun, and H. Zhang. 2014. Investigation of bacterial and fungal diversity in tarag using high-throughput sequencing. J. Dairy Sci. 97:60856096.

Tantikachornkiat, M., S. Sakakibara, M. Neuner, and D. M. Durall. 2016. The use of propidium monoazide in conjunction with qPCR and Illumina sequencing to identify and quantify live yeasts and bacteria. Int. J. Food Microbiol. 234:53-59.

Varhimo, E., K. Savijoki, J. Jalava, O. P. Kuipers, and P. Varmanen. 2007. Identification of a novel streptococcal gene cassette mediating SOS mutagenesis in Streptococcus uberis. J. Bacteriol. 189:5210-5222.

Wang, D., W. Liu, Y. Ren, L. De, D. Zhang, Y. Yang, Q. Bao, H. Zhang, and B. Menghe. 2016. Isolation and identification of lactic acid bacteria from traditional dairy products in Baotou and Bayannur of midwestern Inner Mongolia and q-PCR analysis of predominant species. Korean J. Food Sci. Anim. Resour. 36:499-507.

Watanabe, K., J. Fujimoto, M. Sasamoto, J. Dugersuren, T. Tumursuh, and S. Demberel. 2008. Diversity of lactic acid bacteria and yeasts in Airag and Tarag, traditional fermented milk products of Mongolia. World J. Microb. Biot. 24:1313-1325.

Williams, A. M., and M. D. Collins. 1990. Molecular taxonomic studies on Streptococcus uberis, types I and II. Description of Streptococcus parauberis, sp. nov. J. Appl. Bacteriol. 68:485-490.

Yu, J., W. Gao, M. Qing, Z. Sun, W. Wang, W. Liu, L. Pan, T. Sun, H. Wang, N. Bai, and H. Zhang. 2012. Identification and characterization of lactic acid bacteria isolated from traditional pickles in Sichuan, China. J. Gen. Appl. Microbiol. 58:163-172.

Yu, J., H. Wang, M. Zha, Y. Qing, N. Bai, Y. Ren, X. Xi, W. Liu, B. Menghe, and H. Zhang. 2015. Molecular identification and quantification of lactic acid bacteria in traditional fermented dairy foods of Russia. J. Dairy Sci. 98:5143-5154.

Zhu, H., F. Qu, and L. Zhu. 1993. Isolation of genomic DNAs from plants, fungi and bacteria using benzyl chloride. Nucleic Acids Res. 21:5279-5280. 